Note

\title{
The Benefits of Help in Cooperative Birds: Nonexistent or Difficult to Detect?
}

\author{
Philip A. Downing, ${ }^{1, \star}$ Ashleigh S. Griffin, ${ }^{2}$ and Charlie K. Cornwallis ${ }^{1}$ \\ 1. Department of Biology, Lund University, Lund, Sweden; 2. Department of Zoology, University of Oxford, Oxford, United Kingdom \\ Submitted June 19, 2019; Accepted December 3, 2019; Electronically published April 21, 2020 \\ Online enhancements: supplemental tables. Dryad data: https://doi.org/10.5061/dryad.gmsbcc2j3.
}

\begin{abstract}
A ВSTRACT: In birds that breed cooperatively in family groups, adult offspring often delay dispersal to assist the breeding pair in raising their young. Kin selection is thought to play an important role in the evolution of this breeding system. However, evidence supporting the underlying assumption that helpers increase the reproductive success of breeders is inconsistent. In 10 out of 19 species where the effect of helpers on breeder reproductive success has been estimated while controlling for the effects of breeder and territory quality, no benefits of help were detected. Here, we use phylogenetic meta-analysis to show that the inconsistent evidence for helper benefits across species is explained by study design. After accounting for low sample sizes and the different study designs used to control for breeder and territory quality, we found that helpers consistently enhanced the reproductive success of breeders. Therefore, the assumption that helpers increase breeder reproductive success is supported by evidence across cooperatively breeding birds.
\end{abstract}

Keywords: kin selection, cooperative breeding, indirect fitness, birds, offspring care.

\section{Introduction}

Cooperative breeding has evolved approximately 90 times across the bird radiation (Ligon and Burt 2004; Cornwallis et al. 2017). In this breeding system, adult offspring typically delay dispersal and reproduction to help their parents raise younger siblings (Brown 1987). Hamilton's rule has often been used to explain the evolution of cooperative breeding, as it expresses when a cooperative trait will be favored by selection (Hamilton 1964). It states that helping behavior will spread if $r b>c$, where $r$ is the relatedness between the focal actor ("the helper") and the recipient of the behavior ("the breeder"), $b$ is the fitness benefit to the recipient, and $c$ is the fitness cost to the actor. If

\footnotetext{
* Corresponding author; email: philip.downing@biol.lu.se.

ORCIDs: Downing, https://orcid.org/0000-0002-5286-3153; Griffin, https:// orcid.org/0000-0001-7674-9825.
}

Am. Nat. 2020. Vol. 195, pp. 1085-1091. (C) 2020 by The University of Chicago. 0003-0147/2020/19506-59324\$15.00. All rights reserved.

DOI: $10.1086 / 708515$ both $r$ and $b$ are positive, then helping has the potential to evolve via kin selection because it has a beneficial effect on the fitness of relatives. While an effect of relatedness on the evolution of cooperative breeding has been detected (e.g., Griffin and West 2003; Cornwallis et al. 2010; Green et al. 2016), the fitness benefit of help has largely been taken for granted. This is an important oversight because evidence that helpers increase the reproductive success of the breeders is inconsistent. Helpers increase the reproductive success of breeding pairs in some species (e.g., gray-crowned babbler, Pomatostomus temporalis; Brown et al. 1982), while in other species helpers have little effect (e.g., superb fairy-wren, Malurus cyaneus; Green et al. 1995) or even decrease reproductive success (e.g., rufous vanga, Schetba rufa; Eguchi et al. 2002).

One explanation for the inconsistent evidence for the benefits of help is that they are difficult to measure. Experienced breeders with high reproductive success that occupy good-quality territories are more likely to recruit offspring as helpers, leading to spurious correlations between helping and breeder success (Rowley 1965; Brown et al. 1982). Studies have controlled for breeder and territory quality when measuring the benefits of help using one of three designs: (i) comparison of the reproductive success of the same breeding pair in years when they do and do not have helpers, (ii) experimental removal of helpers, and (iii) multiple regression analysis with breeder and territory quality included as covariates (Cockburn 1998). However, achieving high levels of replication in such controlled studies is difficult, making the null hypothesis that helpers have no effect on breeder reproductive success harder to reject. Furthermore, previous comparative work did not account for breeder or territory quality, potentially resulting in an overestimation of the effects of helpers on breeder reproductive success (Griffin and West 2003).

We conducted a phylogenetic meta-analysis of the effect of helpers on breeder reproductive success (estimates of $b$ ) across cooperatively breeding birds. The detailed data produced by long-term field studies on cooperatively breeding 
birds provide an opportunity to determine whether helper effects on breeder reproductive success are obscured by low statistical power and study design. We focus on studies that separate the effect of helping from the effects of breeder and territory quality on the reproductive success of breeders. We weight evidence according to sample size and account for nonindependence across species due to shared evolutionary history. We explore whether differences in study design (matched-pairs comparisons, removal experiments, and multiple regression) bias estimates of helper effects and discuss the difficulty in measuring the effect of helpers on the survival and future reproduction of breeders (Cockburn et al. 2008; Hammers et al. 2019).

\section{Methods \\ Data Collection}

We only include species in which adult helpers have the potential to gain indirect fitness benefits by increasing the reproductive success of related breeders ( $r b$ in Hamilton's rule). Species in which helpers are reproductively immature were excluded, as our focus is explaining why helping is favored over independent reproduction. We used Riehl's (2013) review of the kin structure of groups of cooperatively breeding birds to identify species that meet these criteria. We then searched Web of Knowledge to identify studies that have measured the relationship between the number of helpers and reproductive success while controlling for the effects of breeder and territory quality. We used the following topic search term: (reproduct* OR group size) AND (binomial species name OR synonym OR English name). We also searched the gray literature (MS and $\mathrm{PhD}$ theses, monographs on individual species, and edited volumes on cooperatively breeding birds) for further studies reporting data. Figure A1 provides an overview of our search strategy, and tables S1-S3 (tables S1-S4 are available online) document which species and studies were included and excluded from our study. The studies from which our effect sizes are extracted assumed that all group members were helpers. All data needed to recreate our analyses, including tables S1-S4 and the supplementary R script (see below), are available in the Dryad Digital Repository (https://doi.org/10.5061/dryad .gmsbcc2j3; Downing et al. 2019).

\section{Effect Size Calculation}

We used Fisher's $Z$-transformed correlation coefficient $(Z r)$ as our effect size (Glass et al. 1981; Koricheva et al. 2013). In our case, this measures the strength of the relationship between helper number and reproductive success:

$$
Z r=\frac{1}{2} \ln \left(\frac{1+r}{1-r}\right)
$$

Positive values of $\mathrm{Zr}$ indicate that helpers are associated with an increase in the reproductive success of breeders, while negative values of $Z r$ indicate that helpers are associated with a decrease in breeder reproductive success. We calculated the sampling variance of each effect size as $1 /(n-3)$, where $n$ is the number of groups studied. References to the exact figures and test statistics from which each effect size was calculated are provided in table S2. Our final sample size included 19 effect sizes from 19 species (fig. A1). Five of these studies used multiple regression to control for breeder and territory quality, three studies used helper removal experiments, nine studies used matchedpairs comparisons (comparing the same breeding pairs in years when they do and do not have helpers), one study used a food supplement experiment, and one study used planned contrasts (pairs and groups on the most productive territories compared). Study design was entered as a four-level fixed effect: matched-pairs comparisons, multiple regression, removal experiments, and "other" (two studies: a food supplement experiment and planned contrasts) in our second statistical model (see below).

\section{Model Construction}

We constructed two random effects meta-analytic models using the MCMCglmm R package to estimate mean effect sizes (Hadfield 2010; R Core Team 2017). We included the following terms in the first model:

$$
y_{i}=\mu+a_{i}+m_{i}+e_{i}
$$

where $y_{i}$ is the effect size $(Z r)$ of species $i, \mu$ is the mean effect size, $a_{i}$ is the phylogenetic effect, $m_{i}$ is the sampling error, and $e_{i}$ is the residual variance. In the second model, we added study design as a fixed effect to assess the impact of different study designs on estimates of $Z r$ :

$$
y_{i}=\mu+\beta X+a_{i}+m_{i}+e_{i},
$$

where $\beta$ is a vector of parameter estimates for each level of our fixed effect and $X$ is the design matrix. This model estimates mean values of our effect size for each level of our fixed effect.

We fitted a phylogenetic (co)variance matrix as a random effect constructed from the phylogenetic trees (Hackett backbone) published by Jetz et al. (2012). To account for phylogenetic uncertainty, we used a sample of 1,300 trees and ran models on each tree for 1,000 iterations, saving the last iteration and discarding the first 999 iterations as a burn-in for each tree. The starting values for the latent variables and variance components for each new tree were taken as the last estimate from the previous tree. We discarded the first 300 trees as a burn-in across trees, giving a total of 1,000 stored iterations, each from a different tree (for further details, see Ross et al. 2013). For random 
effects, we used inverse Wishart priors (variance $=1$ and belief parameter $=0.002$ ).

Parameter estimates reported from these models are the posterior modes and credible intervals (CI) of posterior distributions. Model convergence was assessed by inspecting traces of posterior distributions to evaluate chain mixing, by calculating the degree of autocorrelation between successive iterations in each chain, and by using Gelman and Rubin's convergence test (Gelman and Rubin 1992), which compares within- and between-chain variance (full details are given in the supplementary $R$ script in the Dryad Digital Repository [https://doi.org /10.5061/dryad.gmsbcc2j3; Downing et al. 2019]). To investigate the robustness of our results to Bayesian methods, we also ran each analysis in the metafor $\mathrm{R}$ package using maximum likelihood (Viechtbauer 2010). These are described in the supplementary $\mathrm{R}$ script provided in the Dryad Digital Repository (https://doi.org/10.5061/dryad .gmsbcc2j3; Downing et al. 2019), and the results, which were consistent, are presented in table S4.

\section{Heterogeneity}

We quantified the percentage of variation in $Z r$ attributable to within- and between-study effects and phylogenetic history by dividing estimates of the variance of each term by the total variance in $Z r$. Total variance in $Z r\left(\sigma_{t}^{2}\right)$ was calculated as the sum of within-study variance $\left(\sigma_{m}^{2}\right)$, residual variance $\left(\sigma_{e}^{2}\right)$, and phylogenetic variance $\left(\sigma_{a}^{2}\right)$. Withinstudy variance was calculated as

$$
\sigma_{m}^{2}=\frac{\sum w_{i}(k-1)}{\left(\sum w_{i}\right)^{2}-\sum w_{i}^{2}},
$$

where $w_{i}$ is the inverse sampling variance for species $i$ and $k$ is the number of studies (Nakagawa and Santos 2012). Between-study and phylogenetic variance were estimated from our statistical models. Full results from these models are reported in table S4.

\section{Publication Bias}

To determine whether our sample of effect sizes was biased in a specific direction because of the likelihood of publication in favor of significant results, we visualized funnel plots, conducted a trim-and-fill analysis (Viechtbauer 2010), and used Egger's regression (Egger et al. 1997). Overall, we detected little evidence of publication bias: there was no relationship between the standardized normal deviate of each effect size and its inverse standard error (intercept $=0.43$, $P=.36$; slope $=-0.10, P=.29$ ), and the trim-and-fill analysis suggested that only three studies were missing from the left-hand side of our funnel plot.

\section{Results}

Reproductive success was significantly positively correlated with helper number across 19 species of cooperatively breeding birds in studies that controlled for the confounding effects of breeder and territory quality $(Z r=0.36,95 \%$ $\mathrm{CI}=0.11-0.56$; fig. 1 ; table 1$)$. The overall $Z r$ value of 0.36 translates to a Pearson correlation coefficient of 0.34 , which is considered a medium effect size in evolutionary biology $(<0.3$ are small effect sizes, and $>0.5$ are large effect sizes). In 16 out of 19 species this relationship was positive, indicating that helpers are typically associated with an increase in the reproductive success of breeders in family groups. The relationship between helper number and reproductive success was significantly different from zero in only nine species. Note that for two of these speciesthe white-throated magpie-jay (Calocitta formosa) and the sociable weaver (Philetairus socius) - significant values were reported in the original studies, but our approach of using the number of groups rather than the number of individuals to calculate sampling variances gives nonsignificant values. In 3 out of 19 species - the pinyon jay (Gymnorhinus cyanocephalus), the laughing kookaburra (Dacelo novaeguineae), and the rufous vanga (Schetba rufa) - helper number was negatively correlated with the reproductive success of breeders, but in none of these species was the relationship significantly different from zero.

Study design had a significant impact on estimates of the strength of the relationship between reproductive success and helper number (table 1). When matched-pairs comparisons were used to control for breeder and territory quality by comparing the same breeding pairs in years when they do and do not have helpers, the mean effect size was on average four times lower than when removal experiments or multiple regression was used. In fact, the five lowest estimates of $Z r$ come from studies that used matched-pairs comparisons (fig. 1). However, the two largest effect sizes in our sample - those from the apostlebird (Struthidea cinerea) and the red-cockaded woodpecker (Picoides borealis) - also used this approach. This suggests that matchedpairs comparisons are highly variable when estimating the effect of helpers on reproductive success rather than being consistently low. Higher variability in studies using matchedpairs comparison may be linked to differences in sample sizes, as there were on average two to four times fewer groups studied using this design (mean number of groups: matched-pairs comparisons $=13$, range $=4-24$; removal experiments $=24$, range $=15-37$; multiple regression $=$ 57 , range $=9-153)$.

\section{Discussion}

There is continued debate over the effects of helpers on the reproductive success of breeders in cooperative groups 
Pinyon Jay (matched pairs comparison; $N=16$ groups) Laughing Kookaburra (matched pairs comparison; $N=24$ groups) Rufous Vanga (matched pairs comparison; $N=18$ groups) Brown Jay (matched pairs comparison; $N=8$ groups) Superb Fairy-wren (matched pairs comparison; $N=21$ groups) Florida Scrub Jay (removal experiment; $N=37$ groups) Sociable Weaver (multiple regression; $N=29$ groups) White-fronted Bee-eater (multiple regression; $N=153$ groups) Bicolored Wren (planned contrasts; $N=34$ groups)

Superb Starling (multiple regression; $N=65$ groups) Campo Flicker (multiple regression; $N=27$ groups) White-browed Scrub-wren (matched pairs comparison; $N=9$ groups) White-winged Chough (food supplement experiment; $N=13$ groups) White-throated Magpie-jay (multiple regression; $N=9$ groups) Gray-crowned Babbler (removal experiment; $N=20$ groups) American Crow (matched pairs comparison; $N=5$ groups) Seychelles Warbler (removal experiment; $N=15$ groups) Apostlebird (matched pairs comparison; $N=11$ groups) Red-cockaded woodpecker (matched pairs comparison; $N=4$ groups)

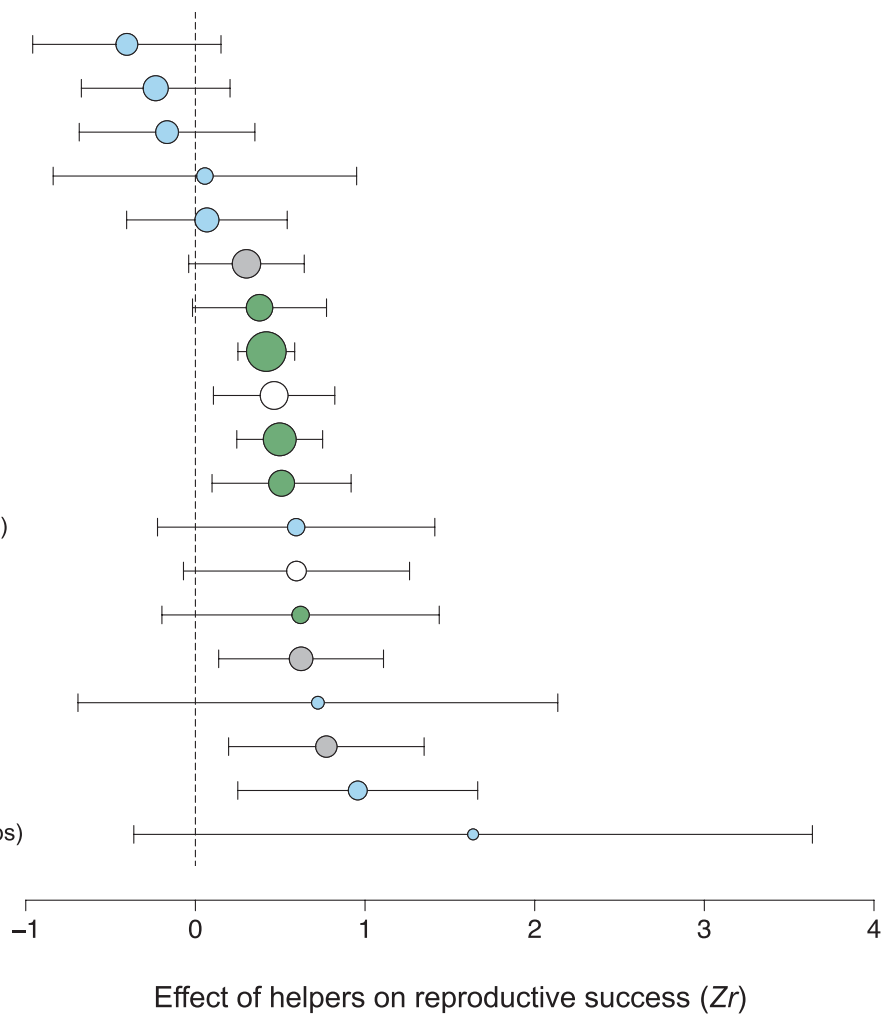

Figure 1: Effect of helpers on breeder reproductive success across cooperatively breeding birds. Fisher's Z-transformed correlation between helper number and reproductive success from studies that controlled for the confounding effects of breeder and territory quality is plotted with $95 \%$ confidence intervals calculated from the sampling variance. Positive correlations indicate that helpers increase the reproductive success of the breeding pair. Circle sizes are proportional to sample sizes, and colors indicate study design.

of birds, raising questions about the contribution of kin selection to the evolution of helping behavior (e.g., Nowak et al. 2010; Queller 2016; Birch 2017). We found clear evidence that helpers enhance the reproductive success of breeders in studies of cooperatively breeding birds that controlled for the effects of breeder and territory quality (fig. 1). In only three species were helpers associated with reduced reproductive success of breeders. However, in all three of these species matched-pairs comparisons were used, and our analysis suggests that this study design produces more variable estimates of the effect of helpers on breeder reproductive success than other study designs (table 1). The general weight of evidence from cooperative birds, therefore, indicates that helpers in family groups typically have a positive effect on breeder reproductive success and that inconsistencies are due to features of study design.

Our finding that helpers increase breeder reproductive success is also relevant for explanations of cooperative breeding based on direct fitness benefits. For example, promoting breeder reproductive success can increase helper fitness if

Table 1: Correlation between helper number and reproductive success (effect size, $\mathrm{Zr}$ ) for the different study designs used to control for the confounding effects of breeder and territory quality

\begin{tabular}{lccc}
\hline Study design & Mean $\mathrm{Zr}$ & 95\% CI & No. species \\
\hline Matched-pairs comparison & .13 & -.20 to .36 & 9 \\
Multiple regression & .43 & .20 to .68 & 5 \\
Removal experiment & .49 & .18 to .86 & 3 \\
Other $^{\text {a }}$ & .59 & .06 to .87 & 2 \\
Mean across species & .36 & .11 to .56 & 19 \\
\hline
\end{tabular}

Note: Parameter estimates are from the phylogenetic meta-analytic models. $\mathrm{CI}=$ credible interval.

a This category includes data from a food supplement experiment and planned contrasts. See table S2 in the Dryad Digital Repository (https://doi.org/10.5061/dryad.gmsbcc2j3; Downing et al. 2019). 
they inherit a larger breeding group or can stay in the group longer, increasing their likelihood of survival, both of which decrease the $c$ term in Hamilton's rule (Kokko et al. 2001, 2002). However, in contrast to kin selection theory, direct fitness benefit explanations for cooperative breeding do not always rely on helpers benefiting breeder reproductive success.

A striking feature of the data presented here is that most studies using controlled designs are underpowered, leading to a failure to reject the null hypothesis of no effect of helpers on breeder reproductive success: only 9 out of 19 studies reported statistically significant effects. Without quantitatively synthesizing these data using meta-analysis, the overall positive effect of helpers on breeder reproductive success would have been missed. Furthermore, the magnitude of helper benefits depended on the method used to control for breeder and territory quality. We found that the estimates of helper benefits from matched-pairs comparisons were highly variable. This may be expected given that studies using this design had smaller sample sizes than studies using multiple regression or removal experiments. Furthermore, cooperative breeders often live in highly variable environments (Jetz and Rubenstein 2011; Cornwallis et al. 2017), making between-breeding-event comparisons problematic, especially as environmental conditions are known to moderate the influence of helpers on reproductive success (Magrath 2001). As matched-pairs comparisons typically cannot account for variation between breeding attempts, obtaining reliable estimates of helper benefits using this approach appears challenging (see also Dickinson and Hatchwell 2004; Cockburn et al. 2008).

The studies in our meta-analysis examined the benefits of help by measuring annual reproductive success. While this is a substantial component of the $b$ term of Hamilton's rule and the most feasible to measure in field studies, it misses any future and "hidden" benefits of helping. For example, in superb fairy-wrens (Malurus cyaneus) helpers have little effect on the number of offspring breeders can raise to independence, but they can increase survival of breeding females by reducing their workload (Cockburn et al. 2008) and investment in eggs (Russell et al. 2007). Consequently, the benefits of help that we have detected here are likely to be even more substantial, but we lack reliable data across species to quantitatively assess this (but see Dixit et al. 2017 for a review of helper effects on egg size and Brouwer et al. 2020 for the importance of group size on survival, including nonlinear helper effects). Furthermore, it is challenging to evaluate the effects of helpers on the future reproduction and survival of breeders, as cooperatively breeding birds are typically long-lived (Arnold and Owens 1998; Downing et al. 2015) and it is difficult to assign future offspring to past helping events (Grafen 1984). One solution has been to study cooperative species that have relatively short life spans, such as the long-tailed tit (Aegithalos caudatus). In this species, the effect of helpers on the future reproduction of breeders has been explicitly included in the estimate of the effect of helpers on breeder fitness, which was positive (Hatchwell et al. 2014).

Field researchers are often faced with significant challenges when it comes to designing studies that control for how conditions vary in natural populations and in gathering sufficient data to detect statistically significant effects. Our results reveal that this has led to an underestimate of the impact that helpers have on offspring production in family groups of cooperatively breeding birds. Without considering effect sizes and statistical power, we would not have been able to accurately evaluate the evidence for a key assumption from kin selection theory - that individuals provide fitness benefits to the recipients of their help. This shows that how we assess evidence matters for the success or failure of a given theory and highlights the dangers of simple vote-counting approaches.

\section{Acknowledgments}

Many thanks to the Swedish Research Council (grant 2017-03880) and the Knut and Alice Wallenberg Foundation (grant 2018.0138) for funding.

\section{APPENDIX}

\section{PRISMA Flowchart}

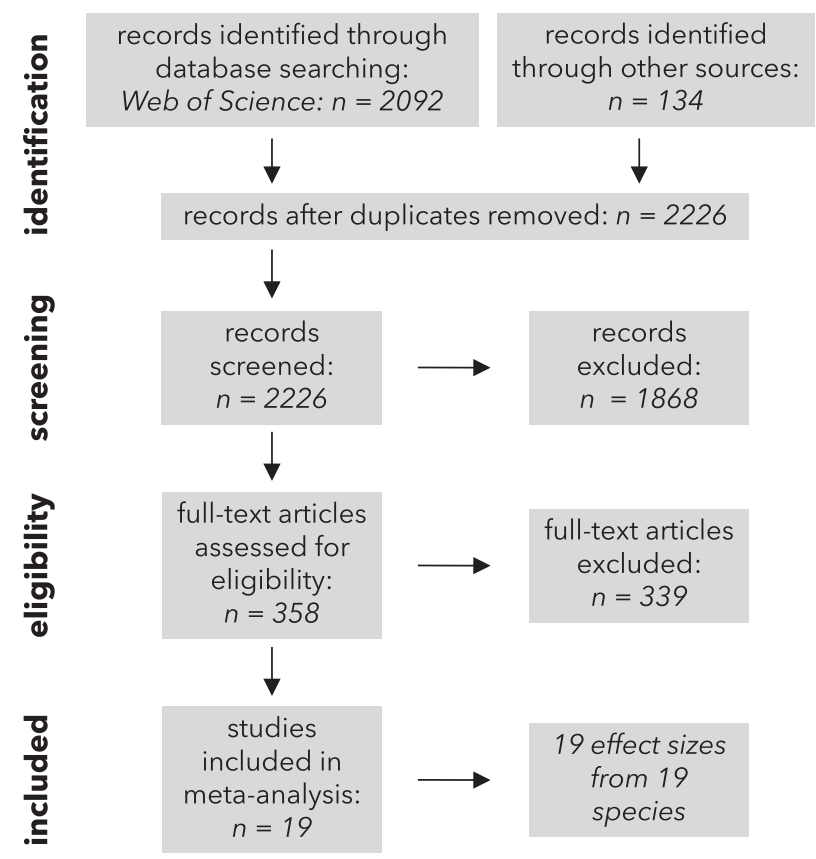

Figure A1: Preferred reporting items for systematic reviews and meta-analyses (PRISMA) flowchart documenting our data collection process (see http://www.prisma-statement.org/). 


\section{Literature Cited}

Arnold, K. E., and I. P. F. Owens. 1998. Cooperative breeding in birds: a comparative test of the life history hypothesis. Proceedings of the Roval Society B 265:739-745.

Birch, J. 2017. The inclusive fitness controversy: finding a way forward. Roval Societv Open Science 4:170335.

Brouwer, L., A. Cockburn, and M. van de Pol. 2020. Integrating fitness components reveals that survival costs outweigh other benefits and costs of group living in two closely related species. American Naturalist 195:201-215.

Brown, J. L. 1987. Helping and communal breeding in birds. Princeton University Press, Princeton, NJ.

Brown, J. L., E. R. Brown, S. D. Brown, and D. D. Dow. 1982. Helpers: effects of experimental removal on reproductive success. Science 215:421-422.

Cockburn, A. 1998. Evolution of helping behavior in cooperatively breeding birds. Annual Review of Ecology and Systematics 29:141177.

Cockburn, A., R. A. Sims, H. L. Osmond, D. J. Green, M. C. Double, and R. A. Mulder. 2008. Can we measure the benefits of help in cooperatively breeding birds: the case of superb fairywrens Malurus cyaneus? Lournal of Animal Ecology 77:430438.

Cornwallis, C. K., C. A. Botero, D. R. Rubenstein, P. A. Downing, S. A. West, and A. S. Griffin. 2017. Cooperation facilitates the colonization of harsh environments. Nature Ecology and Evolution 1:0057.

Cornwallis, C. K., S. A. West, K. E. Davis, and A. S. Griffin. 2010. Promiscuity and the evolutionary transition to complex societies. Nature 466:969-972.

Dickinson, J. L., and B. J. Hatchwell. 2004. The fitness consequences of helping. Pages 48-66 in W. D. Koenig and J. L. Dickinson, eds. Ecology and evolution of cooperative breeding in birds. Cambridge University Press, Cambridge.

Dixit, T., S. English, and D. Lukas. 2017. The relationship between egg size and helper number in cooperative breeders: a metaanalysis across species. PeerI 5:e4028.

Downing, P. A., C. K. Cornwallis, and A. S. Griffin. 2015. Sex, long life and the evolutionary transition to cooperative breeding in birds. Proceedings of the Roval Society B 282:20151663.

Downing, P. A., A. S. Griffin, and C. K. Cornwallis. 2019. Data from: The benefits of help in cooperative birds: nonexistent or difficult to detect? American Naturalist, Dryad Digital Repository, https://doi .org/10.5061/dryad.gmsbcc2j3.

Egger, M., G. D. Smith, M. Schneider, and C. Minder. 1997. Bias in meta-analysis detected by a simple, graphical test. British Medical Journal 315:629-634.

Eguchi, K., S. Yamagishi, S. Asai, H. Nagata, and T. Hino. 2002. Helping does not enhance reproductive success of cooperatively breeding rufous vanga in Madagascar. Iournal of Animal Ecology 71:123-130.

Gelman, A., and D. B. Rubin. 1992. Inference from iterative simulation using multiple sequences. Statistical Science 7:457-511.

Glass, G. V., B. McGaw, and M. L. Smith. 1981. Meta-analysis in social research. Sage, Beverly Hills, CA.

Grafen, A. 1984. Natural selection, kin selection and group selection. Pages 62-84 in J. R. Krebs and N. B. Davies, eds. Behavioural ecology: an evolutionary approach. Blackwell Scientific, Oxford.
Green, D. J., A. Cockburn, M. L. Hall, H. Osmond, and P. O. Dunn. 1995. Increased opportunities for cuckoldry may be why dominant male fairy-wrens tolerate helpers. Proceedings of the Roval Societv B 262:297-303.

Green, J. P., R. P. Freckleton, and B. J. Hatchwell. 2016. Variation in helper effort among cooperatively breeding bird species is consistent with Hamilton's rule. Nature Communications 7:1-7.

Griffin, A. S., and S. A. West. 2003. Kin discrimination and the benefit of helping in cooperatively breeding vertebrates. Science 302:634636.

Hadfield, J. D. 2010. MCMC methods for multi-response generalized linear mixed models: the MCMCglmm R package. Iournal of Statistical Software 33:1-22.

Hamilton, W. D. 1964. The genetical evolution of social behaviour. I. Journal of Theoretical Biology 7:1-16.

Hammers, M., S. A. Kingma, L. G. Spurgin, K. Bebbington, H. L. Dugdale, T. Burke, J. Komdeur, and D. S. Richardson. 2019. Breeders that receive help age more slowly in a cooperatively breeding bird. Nature Communications 10:1-10.

Hatchwell, B. J., P. R. Gullett, and M. J. Adams. 2014. Helping in cooperatively breeding long-tailed tits: a test of Hamilton's rule. Philosophical Transactions of the Roval Society B 369:20130565.

Jetz, W., and D. R. Rubenstein. 2011. Environmental uncertainty and the global biogeography of cooperative breeding in birds. Current Biology 21:72-78.

Jetz, W., G. H. Thomas, J. B. Joy, K. Hartmann, and A. O. Mooers. 2012. The global diversity of birds in space and time. Nature 491:444-448.

Kokko, H., R. A. Johnstone, and T. H. Clutton-Brock. 2001. The evolution of cooperative breeding through group augmentation. Proceedings of the Roval Societv B 268:187-196.

Kokko, H., R. A. Johnstone, and J. Wright. 2002. The evolution of parental and alloparental effort in cooperatively breeding groups: when should helpers pay to stay? Behavioral Ecology 13:291-300.

Koricheva, J., J. Gurevitch, and K. Mengersen, eds. 2013. Handbook of meta-analysis in ecology and evolution. Princeton University Press, Princeton, NJ.

Ligon, D. J., and B. D. Burt. 2004. Evolutionary origins. Pages 5-34 in W. D. Koenig and J. L. Dickinson, eds. Ecology and evolution of cooperative breeding in birds. Cambridge University Press, Cambridge.

Magrath, R. D. 2001. Group breeding dramatically increases reproductive success of yearling but not older female scrubwrens: a model for cooperatively breeding birds? Lournal of Animal Ecology 70:370-385.

Nakagawa, S., and E. S. A. Santos. 2012. Methodological issues and advances in biological meta-analysis. Evolutionary Ecology 26:1253-1274.

Nowak, M. A., C. E. Tarnita, and E. O. Wilson. 2010. The evolution of eusociality. Nature 466:1057-1062.

Queller, D. C. 2016. Kin selection and its discontents. Philosophy of Science 83:861-872.

$\mathrm{R}$ Core Team 2017. R: a language and environment for statistical computing. R Foundation for Statistical Computing, Vienna.

Riehl, C. 2013. Evolutionary routes to non-kin cooperative breeding in birds. Proceedings of the Roval Society B 280:20132245.

Ross, L., A. Gardner, N. Hardy, and S. A. West. 2013. Ecology, not the genetics of sex determination, determines who helps in eusocial populations. Current Biology 23:2383-2387. 
Rowley, I. 1965. The life history of the superb blue wren, Malurus cyaneus. Emu 64:251-297.

Russell, A. F., N. E. Langmore, A. Cockburn, L. B. Astheimer, and R. M. Kilner. 2007. Reduced egg investment can conceal helper effects in cooperatively breeding birds. Science 317:941-944.
Viechtbauer, W. 2010. Conducting meta-analyses in R with the metafor package. Journal of Statistical Software 36:1-48.

Associate Editor: Erol Akçay Editor: Alice A. Winn

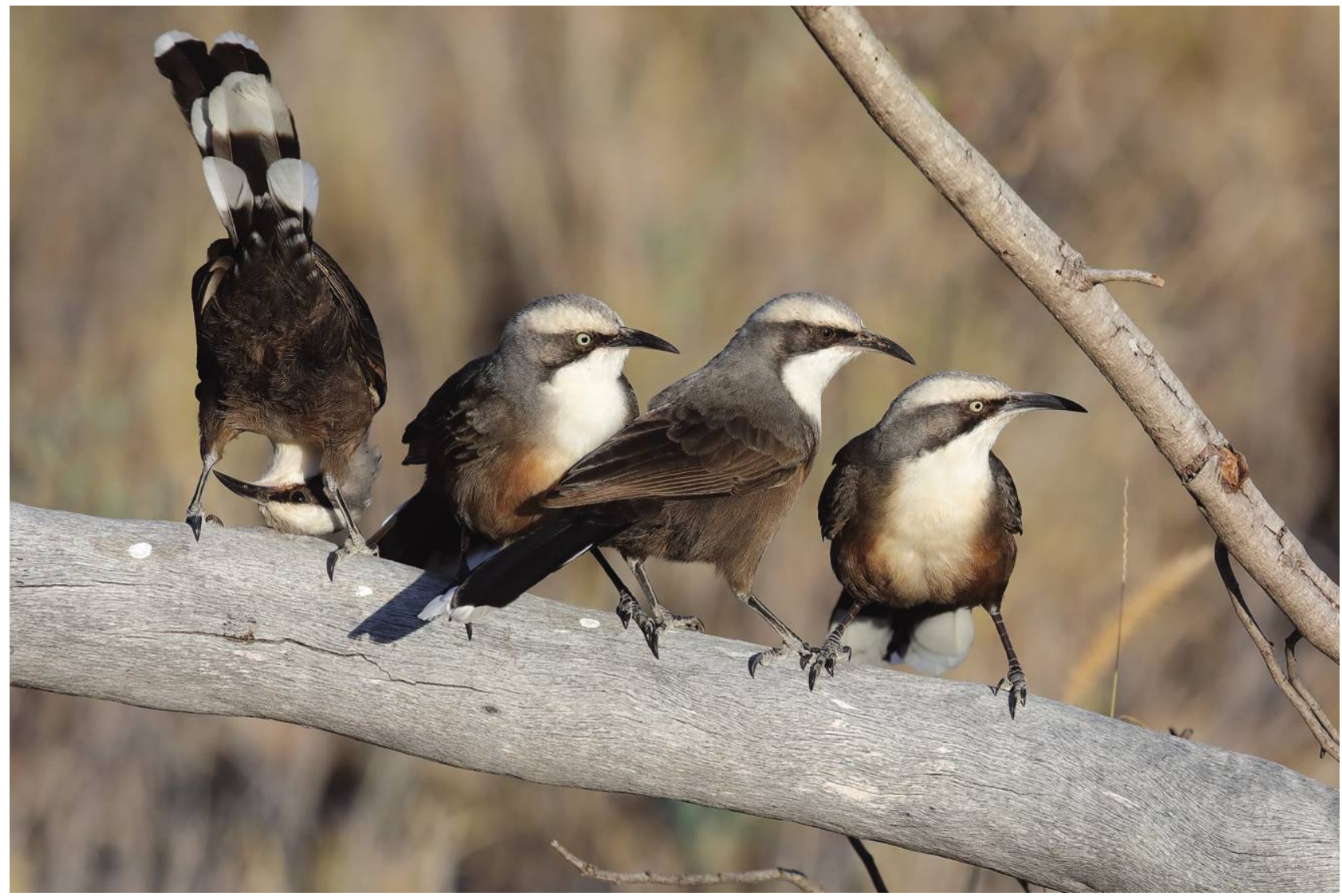

A group of gray-crowned babblers (Pomatostomus temporalis), Epping Forest National Park, Queensland. Photo (c) Graham Lee. All rights reserved. www.flickr.com/photos/grahamlinda_lee/. 